\title{
Quantifying Outcomes in Agricultural Planning
}

\author{
G.M. Lee $^{\text {a }}$, Z. Zhu ${ }^{a}$ and M. Kirby \\ ${ }^{a}$ CSIRO Computational Informatics, CSIRO Clayton, Melbourne, Victoria \\ ${ }^{b}$ CSIRO Land and Water, CSIRO Black Mountain, Canberra, Australian Capital Territory \\ Email: Geoffrey.Lee@csiro.au
}

\begin{abstract}
The availability of ground water to irrigate crops is a key component in food security, particularly in developing regions such as the Indo-Gangetic Basin. Policy settings implemented by governmental authorities can have longer term impact on the livelihoods of farming communities in the region, particularly under the uncertainty of future climate conditions. For example, government policies imposing a minimum level on the ground water table in an agriculture region may result in insufficient ground water available to irrigate a given area of crop, and so the cropped area may have to be reduced consequently.

We have developed a model that computes the cropped area that keeps the water table level above a critical value under the uncertainty of future rainfall scenarios. We use a water balance model to predict the change in water table level caused by growing a fixed area of a particular crop over one year with a given annual rainfall. We then model the annual rainfall as a stochastic process and use Monte Carlo simulations to generate stochastic annual rainfall paths, and adjust the cropped area to maintain the underground water table above a critical level in response to each stochastic annual rainfall path by using the water balance model.
\end{abstract}

We have implemented an optimization procedure that maximises the Sharpe ratio for each year that allows farmers in a region to allocate land to crops in a manner that maximises returns while minimising risk. Starting with land allocations determined through a simple portfolio optimization, we found that considering the effects of rainfall on cropping allocations in addition to accumulating the future cash-flows with a penalty for switching cropping allocations causes a significant difference in cropping allocations when compared to the simple single period optimization scheme.

Our results suggest that the effect of uncertain climate through rainfall in conjunction with certain policy settings may cause a change in optimal cropping land allocations. Further work will focus on developing an optimization model that computes a globally optimal solution, taking into account scenarios where the crop prices do not follow the expected future trajectories.

Keywords: $\quad$ Decision-making under uncertainty, portfolio optimization, stochastic process 


\section{INTRODUCTION}

For food crops, water supply is an essential determinant of their production rates or yields. For food security, decisions on and management of water supply become very important because their impact on future food production can last for many years to come. This is particularly true with regards to ground water supply. The availability of ground water to irrigate crops is a key component in providing food security, especially in developing regions such as the Indo-Gangetic Basin Sikka (2009). In such cases, policy settings promoted by development agencies and implemented by government authorities can impact significantly on the livelihoods of farming communities in the region, particularly under the uncertainty of the climate conditions (Fuss et al (2011), Fuss et al. (2012)).

Imposing a minimum underground water level as a policy may result in a reduction in future crop production if rainfall decreases in the future - there may be insufficient underground water available to irrigate a given area of a certain crop, and so the area of that crop may have to be reduced. The unintended consequences of implementing such a policy should be studied and understood. The amount of groundwater available may depend on the future rainfall, electricity price and crop prices amongst others. However, the future values of these quantities are uncertain and so the decision to invest in a particular technology has to take into account these future uncertainties. This decision-making process is therefore similar to making investment decisions in infrastructure under uncertainty, where significant uncertainties exist when future scenarios need to be considered for 20 or 30 years. In infrastructure investment, real options valuation approach is often relied upon to reach optimal decisions (see Cesena (2011) and Chiara (2007)). In this paper, we quantify and analyze decision-making under uncertainty in agriculture planning when future irrigation water supply is uncertain.

In this paper, we compute the underground water level through a water balance model. Specifically, the knowledge of the change in underground water level caused by cropping a fixed area of crop at a given annual rainfall may allow an adjustment of the cropped area to maintain the water table above a critical limiting value. We use the water balance model developed by Kirby et al. (2010) which determines the water table level for a given rainfall, cropped area and evapotranspiration (ET) value. By utilizing this water balance model, we have developed a model that can compute the average (expected) cropped area that aims to keep the underground water level above a critical value whilst maintaining constant cropped area under the uncertainty of future rainfall.

\section{MODEL}

\subsection{Stochastic Models}

We use two different stochastic models to represent the behavior of future crop prices and the annual rainfall. To model crop prices such as wheat or rice, we use a Geometric Brownian motion model

$d \ln X=\mu d t+\sigma d W$

where $X$ represents a crop price, $W$ is a Wiener process and $\mu$ and $\sigma$ are constants defining the annual drift and volatility of the stochastic process respectively. To model the future annual rainfall, we use an OrnsteinUhlenbeck mean-reverting model

$d \ln X=(\theta-a \ln X) d t+\sigma d W$,

where $X$ represents the annual rainfall, $a$ is the mean-reversion rate, $\theta / a$ is the mean-reversion level and $\sigma$ is the volatility of the stochastic process. The constant parameters defined in Equations (1) and (2) are found through a calibration process described in detail in Zhu (2009). The parameters can be calibrated to historical data, or to different alternative future scenarios.

\subsection{Optimal selection of cropped area}

Without any constraints on water, we assume that farmers will allocate areas of different crops to maximize their income whilst minimizing their exposure to risk. We treat this as a portfolio optimization problem, where the components (assets) of the portfolio are different crops. Using a simple objective such as maximizing the cash flow generated from growing crops would result in a trivial portfolio weighting - the optimal weighting would be to simply crop $100 \%$ of the area with the crop that gives the highest return, irrespective of the uncertainties (risks) in future possible price movement associated with this crop. A rational farmer would balance the risks associated with the various crops by planting a balanced portfolio of crops. 
The objective of such a rational behavior for balancing risks can be mathematically modeled by an objective function that minimizes the risk measure for the crop portfolio. One commonly applied risk measure for constructing optimal portfolios of assets is the Sharpe ratio Sharpe (1994). The Sharpe ratio is the total expected return of the portfolio divided by the portfolio variance, and maximizing this ratio is therefore equivalent to maximizing the risk-adjusted cash-flow, or inversely, minimizing the risk for given expected return. The portfolio found from maximizing the Sharpe ratio is the tangent portfolio to the efficient frontier in Modern Portfolio Theory Markowitz (1952) and this portfolio optimization technique has been widely used in financial asset management Maller (2010). In this paper, we assume uncorrelated risk factors.

\section{Underground water level constraint}

To determine the effect growing a fixed area of a given crop has on the underground water level over time, we utilize a water balance model developed by Kirby et al. (2010). This model consists of three combined balances - surface water, rivers, and the underground water. The principle is to conserve mass, i.e,

Inflows - Outflows + Storage Change $=0$.

The key input parameters are rainfall values and evapotranspiration (ET) values. ET is the water lost by transpiration through plants and evaporation from puddles etc. A more detailed description of the model is presented in Kirby et al. (2010) and Kirby et al. (2013) and also in the appendix to this paper.

We make three key assumptions in order to simplify and implement the water balance model in determining optimal cropping ratios. While the water balance model does include regional variations, we assume each region is identical, with a total area equal to the average regional area. Furthermore, we determine the change in water table level over a period of one year to match the annual rainfall values obtained from our stochastic model, rather than using the monthly resolution in the water balance model. In order to do this, we calculate the average monthly ET and rainfall values (averaged over the 26 years of data provided), which provides an average profile of ET and rainfall for a single year. We fix the ET values for each month to be equal to the 26 year average for that month, whilst still allowing the annual rainfall amount to be stochastic. The annual rainfall amount is distributed monthly according to the calculated average monthly distribution profile.

We then calculate values of the change in underground water level $(\Delta \mathrm{W})$ for different annual rainfall $(\mathrm{R})$ amounts and cropped area (A) for each crop independently, thus generating numerically the function

$\Delta W=f(R, A)$,

for each crop available for cultivation. We note that the function $f$ is not defined explicitly. The change in underground water level is calculated after replicating the annual rainfall over 26 years, allowing the water balance to reach a steady state independent of the initial underground water level. This process generates a look-up table, which, by using numerical interpolation, can be used to find $\Delta \mathrm{W}$ for any given rainfall value and cropped area.

We then use the function defined numerically as written in Equation (4) to maintain the underground water level at or above a critical threshold value by adjusting the cropped area of a given crop to ensure that less ground water is used. Each Monte Carlo trajectory corresponding to the rainfall starts with the same initial cropped area $\mathrm{A}_{0}$, and water table level $\mathrm{W}_{0}$. We then calculate the underground water level $W$ at each (yearly) time-step for each Monte Carlo rainfall trajectory. If we find that the underground water level $W$ will drop below the threshold value $W_{C}$ for a given annual rainfall $R_{i}$, we can adjust the cropped area to find the critical change in underground water level $\Delta W_{C}$ such that $\mathrm{W}=\mathrm{W}_{\mathrm{C}}$, while fixing the annual rainfall to be $\mathrm{R}_{\mathrm{i}}$. That is, we solve the equation:

$f\left(R_{i}, A\right)=\Delta W_{C}$,

for the area A. We restrict the region of area to be non-negative for obvious reasons. We can thus obtain the expected cropped area as a function of time by averaging over the different stochastic trajectories, in addition to the average underground water level. If multiple crops are included, we compute the change in underground water level for each crop independently and sum the results to find the total underground water level $W$. If this total water level drops below the threshold value, we calculate the proportion of the total change in water level caused by each crop. We then adjust the areas of each crop, maintaining the proportions each crop contributes to the new target total water level drop (from the current water table level to the critical value). However, we also require that the total cropped area remains constant. We enforce this condition by increasing the area of the crop that contributes the least to the total drop in water level. As a consequence, the critical water table level is not necessarily strictly achieved, rather the crops that contribute the most to 
decreasing water table is converted to crops that contribute the least. In other words, such system results in the efficient use of water consumption.

\section{CASE STUDY}

The aim of decision-making under uncertainty is to incorporate the uncertainty inherent in future outcomes into decisions taken at the present, producing a set of decision rules that can produce an optimal outcome into the future. In this paper, we aim to find the cropping allocations that optimize the Sharpe ratio under the uncertainties of future crop prices and rainfall. Initial cropping allocations are found by optimizing the Sharpe ratio given that the asset prices follow their expected future paths. The water balance model is utilized to include the effects of uncertain rainfall on the cropping allocations, resulting in different cropping allocations from the optimal portfolio allocation according to Sharp ratio alone. Ideally, a multi-period optimization (Samuelson (1969), Leippold et al. (2004), Çakmak et al. (2006)) should be used to take into account future uncertainty of the crop prices to determine the globally optimal solution including the effects of the rainfall on the cropping allocations. This can be solved via stochastic dynamic programming such as Fuss et al (2011), Fuss et al. (2012); for example, we can use the Least Squares Monte Carlo Longstaff et al. (2001) to find the optimal solution.

In this paper, as a simple illustration, we optimize the Sharpe ratio assuming that the crop price follows its expected mean path in one single scenario. The portfolio returns and variances are accumulated at each time step, beginning from the horizon of 25 years (the time horizon of investment period), stepping backwards in time. We compute a return in the form:

$E\left[R_{T-1}\right]=\sum_{i=1}^{N} w_{i}^{T-1} E\left[R_{i}^{T-1}\right]+E\left[R_{T}\right]$,

where $w_{i}^{T-1}$ is the weighting of the $i$-th crop at time $T-1$ and $E\left[R_{T}\right]$, is the portfolio return at time $T$, and a variance in the form

$\sigma_{T-1}^{2}=\sum_{i=1}^{N}\left(w_{i}^{T-1} \sigma_{i}^{T-1}\right)^{2}+\sigma_{T}^{2}$,

where $\sigma_{i}^{T-1}$ is the standard deviation of $i$-th crop's return at time $T-1$ and $\sigma_{T}^{2}$ is the total portfolio variance at time $T$. We also include a penalty for changing the portfolio allocations, introducing a cost proportional to the current expected return for each asset such that the total return is computed as

$E\left[R_{T-1}\right]=\sum_{i=1}^{N} w_{i}^{T-1} E\left[R_{i}^{T-1}\right]-C E\left[R_{i}^{T-1}\right]\left(\left|w_{i}^{T-1}-w_{i}^{T}\right|\right)+E\left[R_{T}\right]$,

where $C$ is the fractional cost, taken to be 0.25 in this work. We focus on a representative region in Bangladesh that has an area of $2160 \mathrm{~km}^{2}$. We assume that $1500 \mathrm{~km}^{2}(70 \%)$ of this land is available for agricultural activities, and that three crops: rice, wheat and potato can be grown. Over the past 30 years, the average annual rainfall has been approximately $2000 \mathrm{~mm}$. We assume two different future rainfall scenarios; one where the average annual rainfall drops by $1.4 \%$ per annum, reaching a value of approximately $1400 \mathrm{~mm}$ over a 25 year investment period, and a second extreme scenario where the average annual rainfall drops by $3 \%$ per annum, reaching a value of $1000 \mathrm{~mm}$ over the same investment period. We calibrate the Geometric Brownian motion parameters to historical crop prices, obtained from IndexMundi and the US Department of Agriculture. We assume a price volatility of $20 \%$ for each crop. We then develop the cash-flow $V$ in $\$ / \mathrm{km}^{2}$ generated from growing a crop $Y$ by multiplying the yield of crop $Y$ in $\mathrm{kg} / \mathrm{km}^{2}$ by the crop price $P$ in $\$ / \mathrm{kg}$. This cash-flow $V$ is then used to find the expected returns for each crop that is then used in portfolio optimization.

To obtain the cash-flow per unit area after the cropping allocations have been adjusted by the implementation of the water balance, the total cash-flow for a crop $i$

$V_{i}=P_{i} Y_{i} \omega_{i} A_{0}$,

is divided by the original area determined from the simple portfolio optimization. The re-adjustment of cropping allocations is therefore mathematically equivalent to modifying the crops' yields.

\subsection{Initial cropping allocations}

We utilize the numerical optimization procedure to find the expected optimal cropping allocations for the representative region described in Section 3. To provide initial cropping allocations over the 25 year time period as input into the water balance model, we use a simplified optimization procedure where expected returns are not accumulated, and the standard Sharpe ratio is maximized each year assuming the asset prices 
follow their expected future paths and there is no penalty for changing allocations. The returns (cash-flows) and variances from the three crops are determined from 10000 Monte Carlo trajectories.

Figure 1 displays the cropped areas of wheat, rice and potato that optimize the Sharpe ratio over a time period of 25 years. Under the assumptions listed above, the wheat crop should initially be allocated the largest area, but after 13 years the region should transition to growing a majority of rice crop. This is due to the large increase in the variance of the wheat cash income in comparison to the rice crop, as the volatility of the wheat spot price is forecasted to be large in the next 25 years.

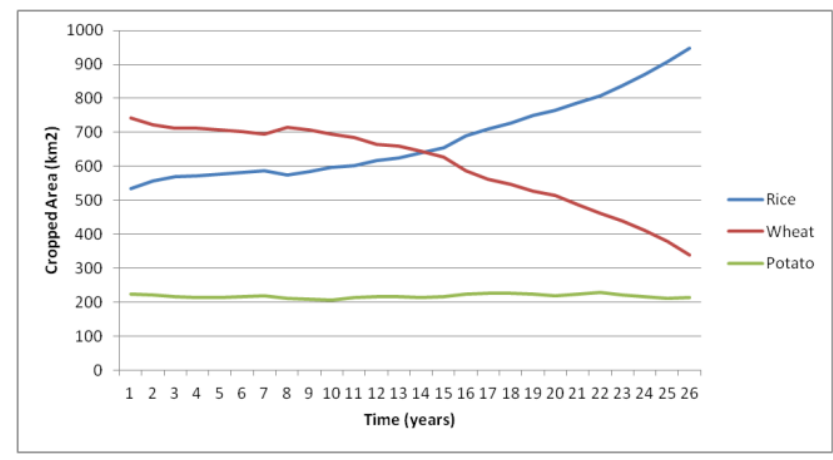

Figure 1. Optimal cropping area allocations for a region that can farm three crops (wheat, rice and potato) obtained from portfolio optimization.

As the future crop prices are unknown, the results indicate the expected optimal portfolios, if the crops' spot prices follow the expected future price precisely. For crop prices modeled using GBM, the difference between the realized optimal portfolio if the crop spot price doesn't follow the expected future price and the results presented here will be small - this won't be the case if a different model such as a mean-reverting model is used.

\subsection{The effect of uncertain future rainfall on optimal cropping allocations}

The introduction of global constraints such as those resulting from climate change policy settings may require alterations to the cropping allocations. To illustrate this, we examine the effects uncertain future rainfall may have on cropping allocations under a policy setting of imposing a limit on the underground water level. Starting with the current optimal crop allocation for each year, we then calculate the amount the underground water level changes at each year using the water balance model described above for each stochastic rainfall trajectory. The water table starts at an arbitrary level of $2 \mathrm{~m}$, and we then set a threshold value of $1.8 \mathrm{~m}$ on the underground water level - implying that the water table level cannot drop by more than $20 \mathrm{~cm}$. The expected cash-flow and variance for each crop were then calculated using the adjusted cropping allocations as per Equation (9). We then carried out the optimization procedure of the Sharpe ratio using the definitions of the portfolio return and standard deviation in Equations (7) and (8). We note that the optimization is carried out each year backwards from the $25^{\text {th }}$ year to today. As there is a penalty to changing the weights in the portfolio from one year to the next, the backward calculated optimal portfolio is optimal locally and with regards to expected future portfolios.

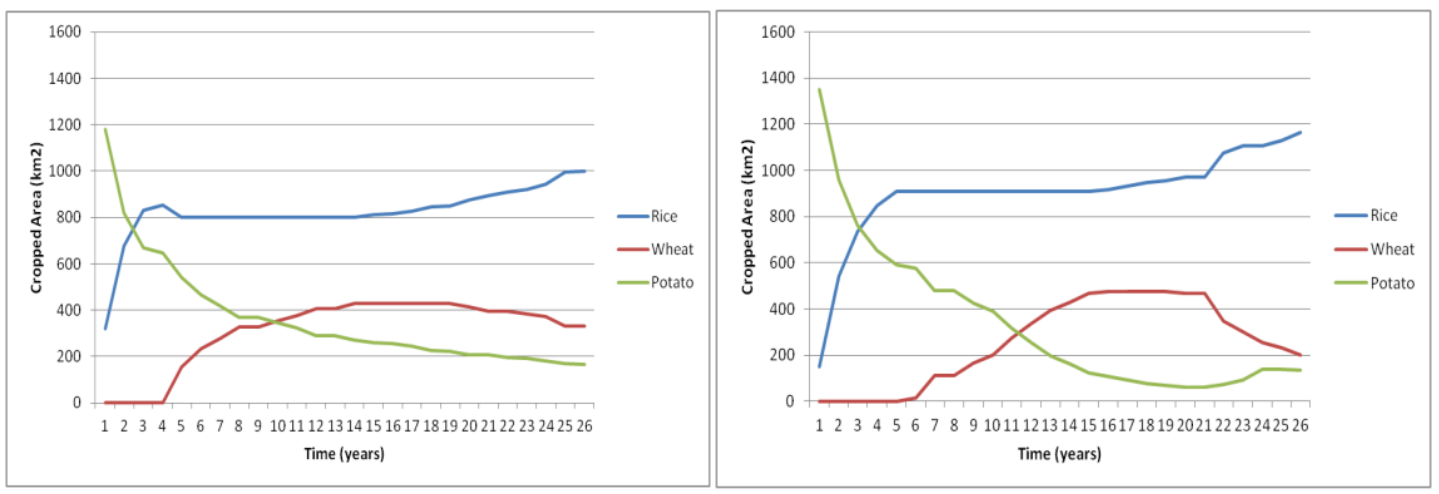

Figure 2. Effect of imposing a limit on water table on the cropped area allocations for different rainfall scenarios. The left plot displays the results for the first rainfall scenario, while the right plot displays the results for the extreme rainfall scenario. 
As the returns and variances are accumulated, the effect on the Sharpe ratio from a single year's returns and variance is reduced, so the decision today tends towards choosing the crop that gives the largest return (cashflow), that being potato. These results vary dramatically from the simple single-period optimization, indicating that incorporating uncertainty is essential in agricultural planning.

\section{CONCLUSIONS AND RECOMMENDATIONS}

We have implemented a model that can adjust cropping areas in response to uncertain future climate conditions to ensure that irrigation water levels remain above a critical threshold value. We used a water balance model to compute the water requirements of crops for a given rainfall and cropped area. Starting with land allocations determined through a simple portfolio optimization, we found that considering the effects of rainfall on cropping allocations in addition to accumulating the future cash-flows with a penalty for switching cropping allocations causes a significant difference in cropping allocations when compared to the simple single period optimization scheme

Our results suggest that the effect of uncertain climate through rainfall in conjunction with certain policy settings can cause a change in optimal cropping land allocations. It would therefore be useful for governments and developmental agencies to conduct quantitative analyses of the impacts certain policy settings have when future climate conditions are uncertain. Further work will focus on developing an optimization model that computes a globally optimal solution, taking into account scenarios where the crop prices do not follow the expected future trajectories.

\section{REFERENCES}

Budyko M.I. (1974), Climate and Life, San Diego: Academic Press.

Çakmak, U. and Özekici, S. (2006). Portfolio optimization in stochastic markets, Mathematical Methods in Operations Research, 63, 151-168.

Chiara, N., Asce, S.M., Garvin, M.J., Asce, M. and J. Vecer (2007). Valuing simple multiple-exercise real options in infrastructure projects, Journal of Infrastrucure Systems, 13(2), 97-104.

Fuss, S., Havlík, P., Szolgayová, J., Schmid, E. and Obersteiner, M. (2011). Large-Scale Modelling of Global Food Security and Adaptation under Crop Yield Uncertainty. EAAE 2011 Congress Change and Uncertainty.

Fuss, S., Havlík, P., Szolgayová, J., Obersteiner, M. and Schmid, E. (2012). Agricultural price volatility under climate change: The impact of multiple objectives on commodity prices, 123rd EAAE Seminar.

IndexMundi, Available online: http://www.indexmundi.com.

Kirby, M., Mainuddin, M. and J. Eastham (2010). Water-use accounts in CPWF basins: Model concepts and description, The CGIAR Challenge Program on Water and Food, Available online: http://www.waterandfood.org/publications/program-publications/working-papers.html.

Kirby, M., Din Ahmad, M-u., Poulton P., Zhu, Z., Lee, G. and M. Mainuddin (2013). Review of water, crop production and system modelling approaches for food security studies in the Eastern Gangetic Plains, CSIRO e-publish EP134291.

Leippold, M., Trojani, F. and Vanini, P. (2004). A geometric approach to multiperiod mean variance optimization of assets and liabilities, Journal of Economic Dynamics and Control, 28, 1079-1113.

Longstaff, F.A and Schwartz, E.S. (2001). Valuing American Options by Simulation: A Simple LeastSquares Approach, The Review of Financial Studies, 14(1), 113-147.

Maller, R.A., Durand, R.B. and Jafarpour, H. (2010). Optimal portfolio choice using the maximum Sharpe ratio, The Journal of Risk, 12(4) 49-73.

Markowitz, H.M. (1952). Portfolio Selection. The Journal of Finance 7(1): 77-91.

Martinez Cesena, E.A. and F.R. Davalos (2011). Evaluation of investments in electricity infrastructure using Real Options and multiobjective formulation, Latin America Transactions (IEEE), 9(5), 767 - 773.

National Agricultural Statistics Service, US Department of Agriculture, Available online: http://www.nass.usda.gov/Statistics_by_State/Washington/Historic_Data/potatoes/potatopr.pdf. 
Samuelson, P.A., (1969), Lifetime Portfolio Selection By Dynamic Stochastic Programming., The Review of Economics and Statistics, 51(3), 239-246.

Sharpe, W.F., (1994). The Sharpe ratio, The Journal of Portfolio Management, 21(1), 49-58.

Sikka, A.K., (2009). Exploring options of participatory water management for livelihood improvements in the Indo-Gangetic Basin, International Journal of River Basin Management, 7(2), 147-155.

Wang Q.J., Pagano T.C., Zhou S.L., Hapuarachchi H.A.P., Zhang L. and D.E. Robertson (2011). Monthly versus daily water balance models in simulating monthly runoff, Journal of Hydrology, 404, 166-175.

Zhang L., Potter N., Hickel K., Zhang Y. and Q. Shao (2008). Water balance modeling over variable time scales based on the Budyko framework - Model development and testing, Journal of Hydrology, 360, $117-131$

Zhu, Z., Graham, P., Reedman, L. and T. Lo (2009). A scenario-based integrated approach for modeling carbon price risk, Decisions in Economics and Finance, 32, 35-48.

\section{APPENDIX:}

A more detailed description of the water balance model used in this work is presented in Kirby et al. (2013). The summary here follows this work.

The water balance model consists of three linked individual balances: the surface, river and ground water balances. Irrigated land is treated separately to non-irrigated land. The water required for irrigation is calculated from the area and crop coefficients of five different crops, including wheat, rice and potato. For each crop in each district in each month, the actual evapotranspiration ET in $\mathrm{mm}, E T_{a}$, is given by:

$E T_{a}=K_{0} E T_{0}$,

where $K_{0}$ is a crop coefficient, and $E T_{0}$ is the reference ET in $\mathrm{mm}$, and $R$ is the rain in $\mathrm{mm}$. The total requirement in bcm (billion cubic metres) for district $i$ in month $j, I R_{i j}$, is given by:

$I R_{i j}=\left\{\begin{aligned} \sum_{k=1}^{n C}\left(E T_{a k i}-R_{i}\right) A_{i k} / 1000000 & , R_{i}<E T_{a k i} \\ 0, & R_{i} \geq E T_{a k i}\end{aligned}\right.$

where $k$ is a crop, $n C$ is the total number of crops, $E T_{a k i}$ is the actual ET of crop $k$ in district $i, A_{i k}$ is the area in $\mathrm{km}^{2}$ of crop $k$ in district $i, \mathrm{R}_{\mathrm{i}}$ is the rain in district $i$, and the factor 1000000 converts from $\mathrm{km}^{2}$ and mm to bcm.

For non-irrigated land, a simple catchment rainfall-runoff model is used to calculate the water balance. The rainfall is partitioned according to the reasoning of Budyko (1974), which applies to average annual runoff, but including a storage that varies monthly. The monthly extension is based on Zhang et al. (2008). A similar model was used by Wang et al. (2011) in modeling Australian catchments, including some in the MurrayDarling basin. The rainfall $P$ is partitioned into runoff $R_{0}$ and infiltration, $I$ ensuring conservation of mass. The infiltration is added to a surface store such as temporary puddles as well as an addition to the soil. The infiltration can be calculated using a Budyko-like equation:

$\frac{I}{\Delta S_{s \max }}=\left(\frac{\left(P / \Delta S_{S \max }\right)^{a_{1}}}{1+\left(P / \Delta S_{S \max }\right)^{a_{1}}}\right)^{1 / a_{1}}$,

where $a_{1}$ is a parameter and $S_{s \text { max }}$ is the maximum capacity of the generalized surface store. Equation (A3) gives the infiltration into the generalized surface store for a given rainfall and value of the parameter $a_{1}$,

The evapotranspiration depends on the potential evapotranspiration, ET pot (the capacity limit), and the surface storage, $S_{S}$ (the supply limit). An equation similar to Equation (A3) above, with a second adjustable parameter, $a_{2}$, is used to model the evapotranspiration. The water balance model proceeds by changing the values of the parameters $S_{s \max }, a_{1}, a_{2}$ and $c_{1}$ until a balance is reached in addition to producing plausible values for variables such as the overall ET and runoff. 